\title{
Der Lagerorganisationsgrad als Steuerungsgröße für optimale Reorganisationszyklen in Kommissioniersystemen
}

\author{
Dipl.-Logist. Daniel Neuhäuser, Prof. Dr.-Ing. Karl-Heinz Wehking \\ Universität Stuttgart \\ Institut für Fördertechnik und Logistik
}

Abstract: Der nachfolgende Artikel beschäftigt sich mit der Lagerreorganisation von

Artikelanordnungen in manuellen Kommissioniersystemen im Lebensmitteleinzelhandel (LEH). Die Kommissionierung erfolgt unter Berücksichtigung der Stabilität der Artikel, eine falsche Einsortierung führt zu höheren Aufwänden im Kommissionierprozess. Die zyklische Neusortierung aller Artikel in die richtige Kommissionier-Reihenfolge wird Lagerreorganisation genannt. Es wird eine Methodik vorgestellt, die die Ermittlung des optimalen Zeitpunktes für eine solche Lagerreorganisation ermöglicht.

\section{$1 \quad$ Einleitung}

Die Versorgung von Verkaufsfilialen im LEH erfolgt in den meisten Fällen durch fest zugeordnete Distributionszentren, die entsprechende Bestellungen der Filialen kommissionieren und versenden. Im Rahmen dieses Artikels wird dabei von einem manuellen Kommissioniersystem (Person-zur-Ware) mit fest zugeordneten Lagerplätzen für die Artikel ausgegangen. Die Herausforderung beim Betrieb eines derartigen Kommissioniersystems liegt in der Kontrolle externer Einflussfaktoren. Dies wird bei der Betrachtung der Entwicklung der Umsatz- und Beschäftigungszahlen deutlich, wie in Abbildung 1 dargestellt.

Es zeigt sich, dass die Verkaufszahlen stark schwanken, insbesondere die Auswirkungen des Weihnachtsgeschäftes können abgelesen werden. Trotz der schwankenden Umsätze bleiben die Zahlen der Beschäftigten weitgehend konstant. Insbesondere die Umsatzschwankungen haben also direkte Auswirkungen auf die Anforderungen an die Distributionszentren, da die Leistungsanforderungen an die Mitarbeiter schwanken. Der Bereich Kommissionierung ist besonderes untersuchungsrelevant, da hier ca. $65 \%$ der Gesamtkosten eines Distributionszentrums bzw. 40\% der Arbeitskosten anfallen. (vgl. [Mup08), [Str10], [Gar05])

Der Optimierungsbedarf wird durch die hohe Artikeldynamik erzeugt: Die feste Lagerplatzzuordnung in einem manuellen System erfordert eine Anordnung der Artikel gemäß der optimalen Kommissionierreihenfolge. Vereinfacht heißt dies, dass schwere und stabile vor leichten bzw. empfindlichen Artikeln kommissioniert werden müssen.

Da ein häufiger Wechsel der Artikel stattfindet, kann nicht garantiert werden, dass die neuen Artikel an der richtigen Position einsortiert werden. Dies führt zu erhöhten 
Zeitbedarfen für das Umpacken von Artikel auf dem Kommissionierladungsträger und damit zu einer Verringerung der Systemleistung.

\section{Umsätze und Beschäftigte im Einzelhandel}

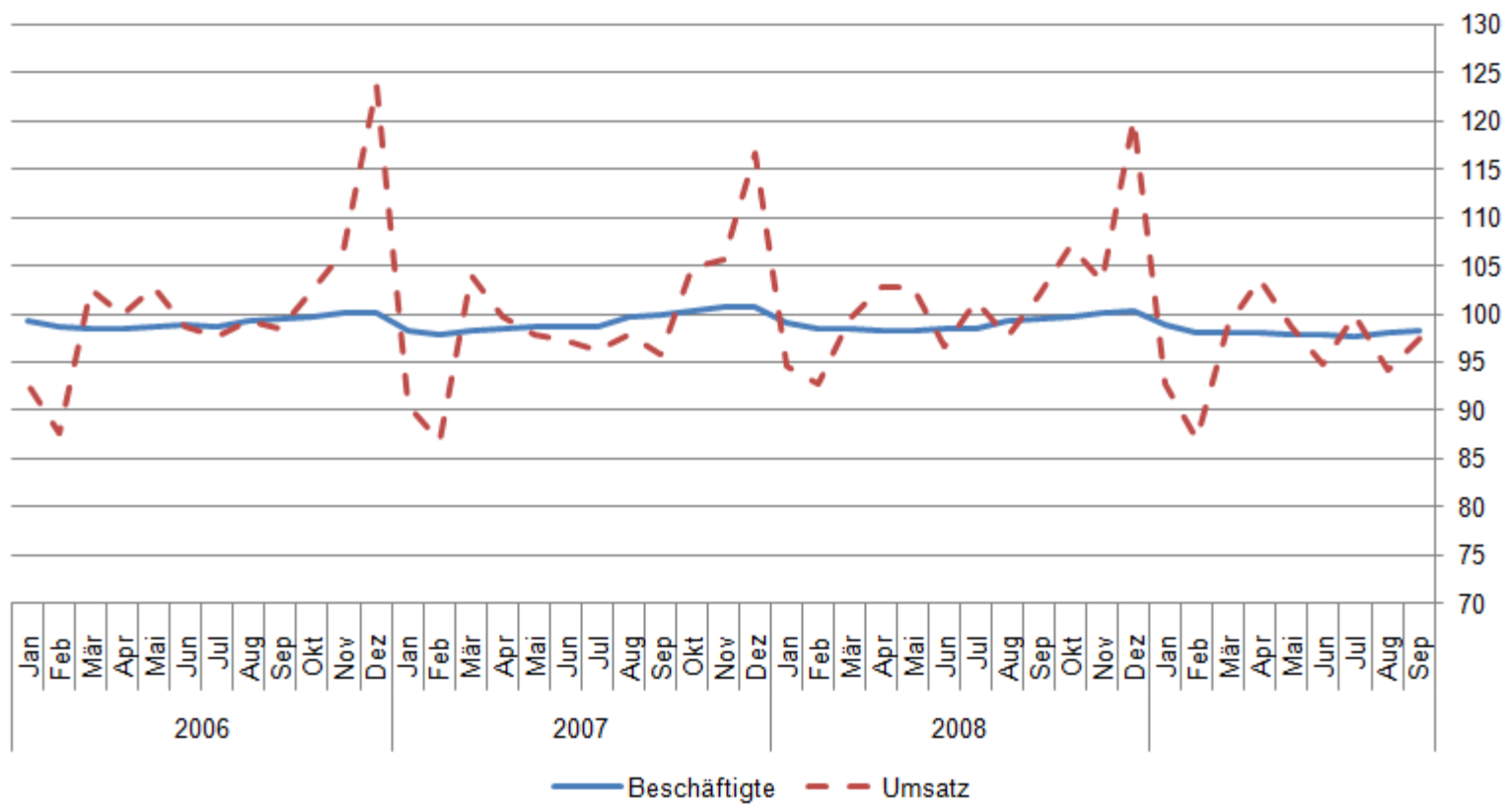

Abbildung 1: Umsätze \& Beschäftigte im Einzelhandel ${ }^{1}$, Angaben in Prozent, Referenzjahr 2005

\section{$2 \quad$ Stand der Forschung}

Gemäß VEITINGER sind Reorganisationsprozesse Ereignisse, die wiederholt im Lebenszyklus von Unternehmen auftreten. $\mathrm{Zu}$ den Kriterien von Reorganisationsmaßnahmen gehören auch, dass die Eingriffe nicht alltäglich sind und weitreichende Auswirkungen auf die Mitarbeiter haben (vgl. [Vei97]).

Eine weitergehende Unterscheidung ist möglich, wenn die unterschiedlichen Entscheidungsebenen in einem Distributionszentrum betrachtet werden. Die Planung und Steuerung eines Distributionszentrums unterteilt sich damit in drei Ebenen, in denen Entscheidungen getroffen werden müssen. Dies umfasst die strategische, die taktische und die operative Ebene; Investitionskosten fallen im strategischen und taktischen Bereich an, in der operativen Ebene werden die strategischen und taktischen Vorgaben umgesetzt. Die Zuordnung von Artikeln zu Lagerplätzen ist Bestandteil der operativen Ebene. (vgl. [Rou00])

Die Reorganisation der Artikelanordnung ist also eine operative Form von Reorganisationsmaßnahmen in Distributionszentren. Für diese Form sind zwei Probleme zu lösen und zu bewerten: Zuerst muss jedem Artikel der richtige Lagertyp und anschließend der optimale Lagerplatz zugewiesen werden. Darüber hinaus müssen die Auswirkungen der Artikelanordnung auf die Kommissionier- und

\footnotetext{
${ }^{1}$ Stand: 22.11.2009; (Statistisches Bundesamt, 2009). 
Nachschubleistung bewertet werden. Der Stand der Forschung für diese Probleme wird nachfolgend kurz diskutiert.

\subsection{Lagerplatzzuordnung}

Das Problem der Lagerplatz-Zuordnung (Storage-Location Assignment-Problem; SLAP) als operatives Problem in der Steuerung von Distributionszentren ist definiert als Menge von Regeln, welche die Zuordnung von Artikeln zu Lagerplätzen kostenoptimal ermöglichen (vgl. [Wäs04], [Kos07]). Dabei soll mit gegebenen Informationen zum Lagerbereich (dies umfasst Lagertechniktechnik und -layout sowie Anzahl, Dimensionierung und Verfügbarkeit der Lagerplätze), zur Artikelstruktur (also Abmessungen, Mengen etc.) sowie (Kommissionier-)Strategien (vgl. [Cha11]) die Zuordnung zwischen Lagerplatz und Artikel unter Berücksichtigung von Leistungs- und Kompatibilitätsrestriktionen durchgeführt werden (vgl. [Gu07]). Diese Restriktionen umfassen die Parameter der Artikel (Abmessungen, Gewicht, Lieferdatum, ...) sowie die Attributen der Lagerplätze (Dimensionen, Temperatur, Entfernung zur Ein- und Auslagerposition etc.).

Die vielfältigen Unterscheidungsmöglichkeiten sind in Abbildung 2 dargestellt:

\begin{tabular}{|c|c|c|c|c|}
\hline \multicolumn{5}{|c|}{ Unterscheidungsmöglichkeiten Lagerplatzzuordnung } \\
\hline Informationsverfügbarkeit & \multicolumn{2}{|c|}{ statisch } & \multicolumn{2}{|c|}{ dynamisch } \\
\hline Vorhandene Informationen & keine & Stammdaten & Bewegungsdaten & Prognosen \\
\hline Anzahl Lagertyp & \multicolumn{2}{|c|}{1} & \multicolumn{2}{|c|}{$\mathrm{n}$} \\
\hline Art Lagertechnik & \multicolumn{2}{|c|}{ automatisch } & \multirow{2}{*}{\multicolumn{2}{|c|}{$\frac{\text { kombiniert }}{\mathrm{n}}$}} \\
\hline Zonung & \multicolumn{2}{|c|}{1} & & \\
\hline Klassen & \multicolumn{2}{|c|}{1} & \multicolumn{2}{|c|}{$\mathrm{n}$} \\
\hline Klassenkriterium & $\mathrm{COI}$ & $\mathrm{ABC}$ & Geometrie & kombiniert \\
\hline Optimierungskriterium & Auslastung & Wegstrecke & Kosten & Durchlaufzeit \\
\hline Nachschub & \multicolumn{2}{|c|}{ separat } & \multicolumn{2}{|c|}{ integriert } \\
\hline
\end{tabular}

Abbildung 2: Unterscheidungsmöglichkeiten Lagerplatzzuordnung

Die Lösung eines SLAP ist in verschiedene Problemklassen und Entscheidungsmöglichkeiten unterteilt. Generell gilt jedoch, dass sowohl die Komplexität der Lagerplatzzuordnung als auch die der Lagertyp-Zuordnung np-hart ist (vgl. [Luo09]) und daher im Allgemeinen nur ein heuristischer bzw. mehrstufiger Lösungsalgorithmus praktikabel ist (vgl. [Abd94]). In manuellen Systemen werden hier im Wesentlichen Paletten, Durchlauf- und Fachbodenregale verwendet (vgl. [Gra92]). Dies konkretisiert VICKSON mit der Unterteilung des Problems in zwei Ebenen: Zuerst müssen Artikel verschiedenen Lagertypen zugeordnet werden und dann auf unterschiedliche Plätze verteilt werden (vgl. [Vic97]).

\subsection{Bewertung optimale Wegzeit}

Allgemein hängen die Leistungsfähigkeit und Effizienz eines Distributionszentrums von der optimalen Zusammenstellung des Layouts, Lager- und Nachschubplanung sowie der Kommissionier- Lager- und Nachschubstrategie ab (vgl. [Gra92]). ROUWENHORST erweitert diese Definition um Kriterien: Dies sind Invest- und (c) 2011 Logistics Journal : Proceedings - ISSN 2192-9084 
Betriebskosten, Flexibilität, Durchsatz, Kapazität. Durchlaufzeiten und Qualität der Kommissionierung. Wesentliches Bewertungskriterium für Distributionszentren (Typus Auslieferungszentrum) ist der maximale Durchsatz mit minimalen Kosten (vgl. [Rou00]).

Dies umfasst viele Faktoren, bezüglich der Lagerstrategie (Lagerplatzzuordnung, Kriterien für die Bildung von Artikelklassen) (vgl. [Ren08]) sowie der Batching- und Routing-Strategie. Zusätzlich sind Lagerstrategie- und Routing-Strategie abhängig voneinander. (vgl. [Pet99])

Diese Abhängigkeit wird anhand der Veränderung der durchschnittlichen Wegstrecke bei der Kombination dieser beiden Strategiekomponenten beschrieben. Eine ähnliche Abhängigkeit existiert zwischen der Lagerstrategie und Größe einer Kommissionierzone bzw. der Anzahl Klassen (vgl. [Rat99]). Zusätzlich hat die Effizienz bzw. Leistungsfähigkeit eines Distributionszentrums direkten Einfluss auf die Gesamtleistung einer Supply Chain (vgl. [Ren08]).

Die durchschnittliche Weglänge hängt stark linear von der Wegzeit ab, daher ist die Optimierung der durchschnittlichen Weglänge eines Kommissioniersystems ein häufiges Kriterium (vgl. [Pet99b]). Eine Untersuchung über die Auswirkungen von Artikelanordnungsmustern hat DÖRNER durchgeführt: Ein gängiges Optimierungskriterium ist die Minimierung der Wegstrecke für die Abarbeitung der Kundenaufträge (vgl. [Doe04]).

Es zeigt sich, dass als relevantes Bewertungskriterium im Allgemeinen die Reduzierung der Wegstrecke herangezogen wird (vgl. [Gu05]). Für den hier untersuchten Fall ist das nicht sinnvoll, eine signifikante Wegereduktion durch die Artikelanordnung kann nicht realisiert werden. Vielmehr muss hier eine Methodik entwickelt werden, die die Kommissionierzeit unter Berücksichtigung von Störgrößen wie Umpacken betrachtet.

\section{Berechnung optimaler Reorganisationszyklen}

Nachfolgend soll eine Methodik vorgestellt werden, die unter der Integration von Umpackzeiten in Abhängigkeit der aktuellen Artikelanordnung eine Bewertung von Reorganisationsprozessen ermöglicht. Aufbauend auf einer formalen Definition für die Bewertung falsch einsortierter Artikel über einen Lagerorganisationsgrad wird eine Formel entwickelt, die die Berechnung von Reorganisationszyklen ermöglicht.

\subsection{Definition Lagerorganisationsgrad}

Die formale Beschreibung des Organisationgrades eines Lagers ist eine wesentliche Stufe für die Bewertung der Systemleistung und der Detektion einer erforderlichen Lagerreorganisation.

Die Bewertung des Organisationsgrades impliziert, dass eine optimale ArtikelAnordnung bekannt ist, jede Einlagereinheit E (im Allgemeinen ein Ladungsträger, 
der einen Lagerplatz belegt) muss einer optimalen Lagerzone zugeordnet werden. Für die sinnvolle Anwendung eines Organisationsgrades muss gleichzeitig die Situation entstehen können, dass ein Artikel außerhalb seiner optimalen Lagerzone eingelagert wird (vgl. [Gla05]). Dies schränkt wiederum die möglichen Betriebsstrategien ein, anwendbar ist der Lagerorganisationsgrad also in zonen- oder klassenbasierten Lagerstrategien, mit einer hohen Dynamik. Es kann also ein häufiger Wechsel der Einlagereinheiten erfolgen.

Für ein Lager, welches die oben beschriebenen Anforderungen erfüllt, kann der Lagerorganisationsgrad hergeleitet werden:

Für eine spezifische Lagereinheit $E_{i}$, die zur Klasse $j$ gehört, existiert ein optimaler Lagerbereich $L_{\text {opt }}$. Aus organisatorischen Gründen (Platzmangel, etc.) ist der reale Lagerplatz in einem falschen Bereich und wird mit $L_{\text {Real }}$ bezeichnet. Damit kann die Veränderung der Anordnung berechnet werden:

$\rho=\left|L_{\text {opt,i }}-L_{\text {Real,i, }}\right|^{\alpha_{j}}$

Formel 3.1-1: Änderung Artikelanordnung

Unter der Annahme, dass die Lagerbereiche durchgehend nummeriert sind, kann über Formel 3.1-1 der Abstand vom realen zum optimalen Lagerbereich bestimmt werden.

Der Faktor $\alpha_{j}$ gewichtet diesen Wert in Abhängigkeit von der Artikelklasse. Für Klassen mit einer hohen Zugriffshäufigkeit führt ein Wert von $\alpha>1 \mathrm{zu}$ verstärkten Auswirkungen der falschen Einordnung. Der Bezug zu einem Gesamtsystem stellt sich über Formel 3.1-2:

$$
\mathrm{L}_{\text {org }}=\frac{\text { Anzahl Artikel }}{\text { Anzahl Artikel }+\sum_{\mathrm{i}=1}^{n} \mid \mathrm{L}_{\mathrm{opt,i}}-\mathrm{L}_{\text {Real, },\left.\right|^{\alpha_{j}}}}
$$

Formel 3.1-2: Lagerorganisationsgrad

Der Lagerorganisationsgrad ist also die Summe der falsch eingeordneten Artikel über alle Artikel. Damit ist ein System optimal konfiguriert, wenn der Lagerorganisationsgrad gegen 1 konvergiert. Dies entspricht dann dem energieminimalen Zustand, da keine Lagereinheit seinen aktuellen Lagerbereich verlassen möchte. Damit kann auch das formale Problem zur Steuerung des Lagersystems formuliert werden, welches den Lagerorganisationsgrad gegen das Maximum 1 konvergieren lässt:

$$
\frac{\text { Anzahl Artikel }}{\text { Anzahl Artikel }+\sum_{\mathrm{i}=1}^{n}\left|\mathrm{~L}_{\text {opt,i }}-\mathrm{L}_{\text {Real,i, }}\right|^{\alpha_{\mathrm{j}}}} \rightarrow 1
$$

Formel 3.1-3: Problemformulierung

Es ist an dieser Stelle sinnvoll, die Aussage des Lagerorganisationsgrades zu konkretisieren. Für die manuell bediente Kommissionierung (Mann-zur-Ware) bedeutet eine hohe Organisation eine gute Qualität der kommissionierten Palette; das Sortierkriterium zur Bestimmung des optimalen Bereiches enthält im 
Allgemeinen auch Kriterien bezüglich der optimalen Kommissionierreihenfolge. Bezüglich der Organisation des Nachschublagers bedeutet eine Verbesserung eine Verkürzung der Nachschub-Transportwege. Für die zu Beginn der Arbeit beschriebene Problematik der Lagerreorganisation können also zwei wesentliche Punkte erkannt werden: Ein niedriger Organisationsgrad in der Kommissionierung führt zu einem suboptimalen Betrieb des Kommissionierbereiches. Eine Verbesserung der Organisationsgrade führt also zu einer Optimierung von Kommissionier- und Nachschubleistung, verursacht aber Kosten durch erforderliche Umlagerungen und entsprechende Planungsaufwände. Dies ist gleichzeitig auch das Zielproblem der Lagerreorganisation.

\subsection{Ableitung funktionaler Zusammenhänge}

Die Ermittlung eines optimalen Reorganisationszeitpunktes erfordert die Beschreibung der Abhängigkeit der Zeitbedarfe für Kommissionierung und Nachschub. Um dies zu ermitteln wurde eine Simulationsmethodik implementiert, die durch Objektklassen und Datenstrukturen die flexible Modellierung und Simulation von manuellen Kommisssionierbereichen ermöglicht. Anhand dieser Methodik wurden in ca. 500 Simulationsläufen etwa 1,5 Mio. Kommissionierpositionen simuliert. In den Simulationsläufen wurden die Artikelanordnung, die Zuordnung zu Lagertypen und der Lagerorganisationsgrad variiert. Die Unordnung des Kommissionierbereiches verändert sich also stetig, die Auswirkungen auf die Kommissionierleistung ergibt sich durch die Modellierung von Strafzeiten, die von der Reihenfolge der kommissionierten Artikel abhängig ist.

Abbildung 3 zeigt eine beispielhafte funktionale Approximation für die Gesamtzeit pro Kommissionierposition ( $T$ _pos, y) in Abhängigkeit vom Lagerorganisationsgrad (x). Es zeigt sich, dass hier ein linearer Zusammenhang besteht mit einem guten Bestimmtheitsmaß $\left(R^{2}\right)$.

Die Methodik ist analog für die Zeitabhängigkeit von Nachschub und Lagerorganisationsgrad, dies wird allerdings an dieser Stelle nicht diskutiert. Damit können die wesentlichen Kosten funktional beschrieben werden. Bezeichnet man $\mathrm{K}(\mathrm{x})$ als Kostenfunktion für die Kommissionierung und analog $\mathrm{N}(\mathrm{x})$ als Kostenfunktion des Nachschubs, beide in Abhängigkeit vom Lagerorganisationsgrad, so bildet sich die gesamte Kostenfunktion $\mathrm{K}^{*}(\mathrm{x})$ über:

$$
\mathrm{K}^{*}(\mathrm{x})=\mathrm{K}(\mathrm{x})+\mathrm{N}(\mathrm{x})
$$

Formel 3.2-1: Kostenfunktion gesamt

Eine Detaillierung dieser Kostenfunktionen erfolgt an dieser Stelle nicht.

Nachfolgend müssen dann die entstehenden Kosten für die Durchführung ebenfalls in Abhängigkeit vom Lagerorganisationsgrad beschrieben werden. Eine Lagerreorganisation besteht aus zwei Komponenten: Administrativ muss festgelegt werden, in welcher Reihenfolge die Artikel angeordnet werden müssen; daraus leiten 
sich konkrete Umlagerungsaufträge ab. Die operative Bearbeitung dieser Aufträge bildet dann den physischen Teil einer Reorganisationsmaßnahme ab.

\section{Verhältnis T_pos zu Lagerorganisationsgrad}

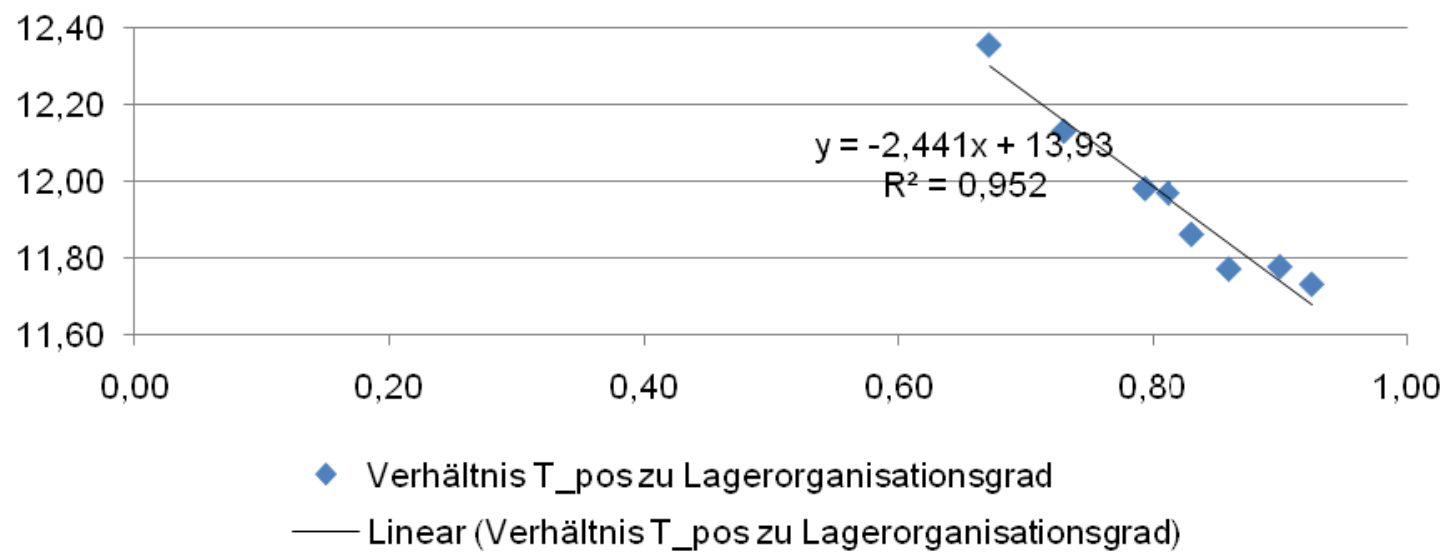

Abbildung 3: Funktional t_pos zu Lagerorganisationsgrad

Aus der Definition des Lagerorganisationsgrades (vgl. Formel 3.1-2) kann zurückgerechnet werden, wie viele Umlagerungen für die Verbesserung des aktuellen Reorganisationsgrades auf den Zielorganisationsgrad erforderlich sein.

Für eine vereinfachte Betrachtung kann der Term $\sum_{i=1}^{n}\left|L_{\text {opt,i }}-L_{\text {Real, }}\right|^{a_{j}}$ durch $L_{G e s}$ ersetzt werden. Die Kosten für eine Reorganisation sind abhängig von der Anzahl der fehlerhaft eingelagerten Artikel, also von der Höhe von $\mathrm{L}_{G e s}$. Um die Anzahl Umlagerungen für den Wechsel von Lagerorganisationsgrad $x_{1}$ auf $x_{2}$ zu berechnen, muss entsprechend umformuliert werden.

$$
x=\frac{\text { Anzahl Artikel }}{\text { Anzahl Artikel }+L_{\text {Ges }}}
$$

Nach Umstellung kann die Menge der erforderlichen Umlagerungen $L_{\text {Reorg }}$ für den Wechsel vom aktuellen Zustand $\mathrm{x}_{1}$ auf den Ziel-Organisationsgrad $\mathrm{x}_{2}$ beschrieben werden:

$\mathrm{L}_{\text {Reorg }}=$ Anzahl Artikel $* \frac{1-\mathrm{x}_{2}}{\mathrm{x}_{2}}-$ Anzahl Artikel $* \frac{1-\mathrm{x}_{1}}{\mathrm{x}_{1}} \quad \begin{array}{r}\text { Formel 3.2-3: Um- } \\ \text { stellung zu } \mathrm{L}\end{array}$

Es muss allerdings berücksichtigt werden, dass diese Kennzahl nicht eindeutig die Anzahl der falsch eingelagerten Artikel beschreibt, vielmehr ist dies die Anzahl der Klassen, die gewechselt werden müssen.

Unter Berücksichtigung eines Zeitwertes $t_{k}$ für die Umlagerung eines Artikels in die nächsthöhere Klasse können die anfallenden Kosten abgeschätzt werden. Wenn ein Artikel mehrere Klassen umgelagert werden muss, fällt diese Zeitdauer 
dementsprechend häufiger an. Ergänzt um den Kostensatz pro Zeiteinheit $k_{R}{ }^{*} t_{k}$ können die mengenabhängigen Kosten für eine Reorganisationsmaßnahme berechnet werden:

$\mathrm{K}_{\text {Reorg }}=\mathrm{L}_{\text {Reorg }} * \mathrm{~K}_{\mathrm{R}} * \mathrm{t}_{\mathrm{k}}$

Formel 3.2-4: Berechnung Kosten Reorga

Hier ist allerdings nicht berücksichtigt, welcher organisatorischer Aufwand für die Abwicklung einer solchen Reorganisationsmaßnahme anfällt, sozusagen als mengenunabhängiger Kostensatz. Der Parameter $t_{k, K o n s t}$ bildet die Kosten ab, die für Planung und Vorbereitung anfallen. Dieser Aufwand ist zwar in gewisser Weise auch mengenabhängig (je größer die Artikelanzahl ist, desto höher ist der Aufwand), allerdings ist der wesentliche Anteil artikelunabhängig. Damit können die Gesamtkosten formuliert werden:

$\mathrm{K}_{\text {Reorg, Ges }}=\Delta \mathrm{K}_{\mathrm{K}}+\Delta \mathrm{K}_{\mathrm{N}}-\left(\mathrm{L}_{\text {Reorg }} * \mathrm{~K}_{\mathrm{R}} * \mathrm{t}_{\mathrm{k}}+\mathrm{t}_{\mathrm{K}, \text { Konst }}\right)$

Formel 3.2-5: Berechnung Kosten Reorga

Aufbauend auf Formel 3.2-5 können nun diese Kosten mit den Nutzenpotentialen aus Formel 3.2-1 zusammengeführt werden.

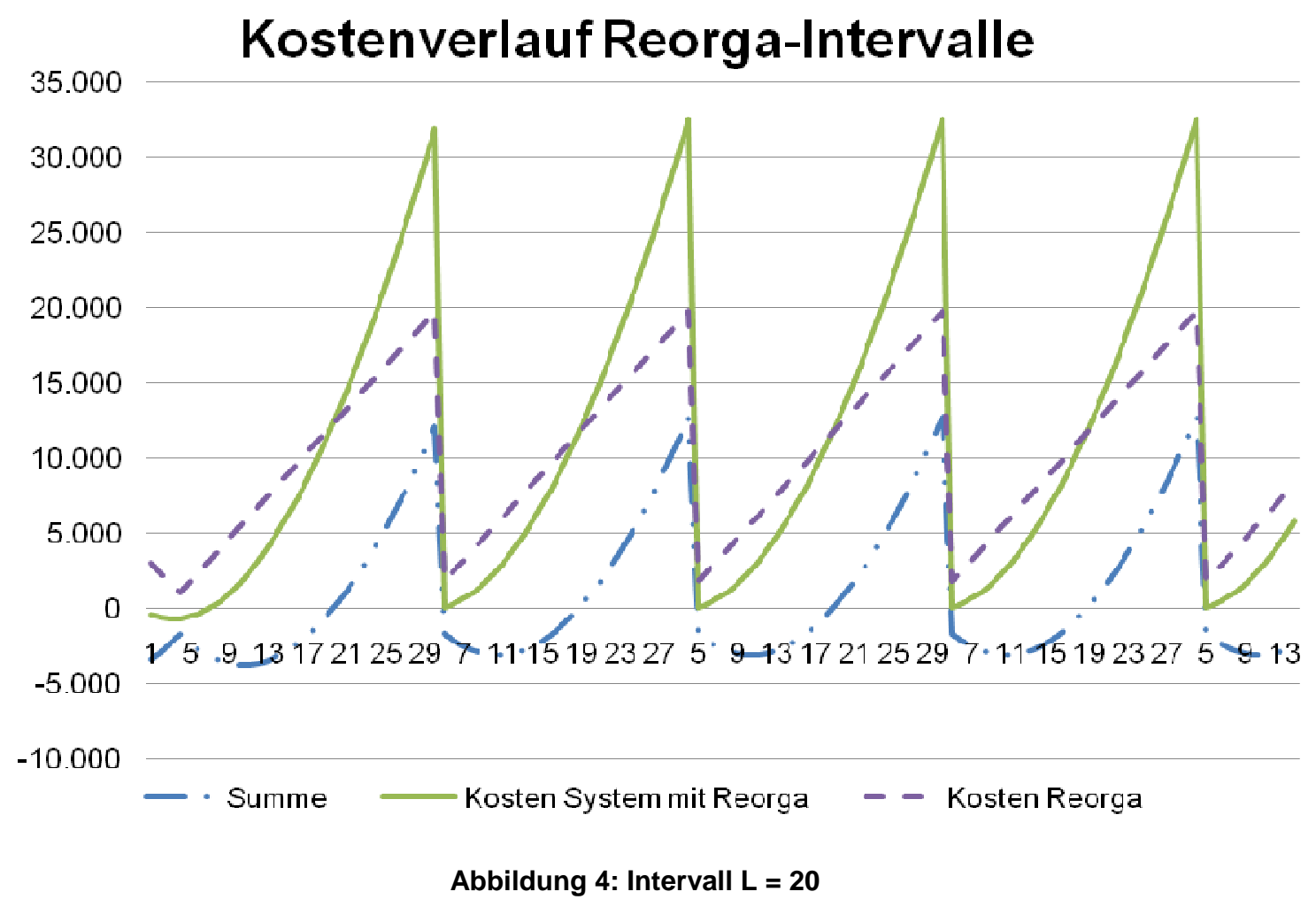

Abbildung 4 zeigt den Verlauf der aus Simulationsexperimenten approximierten Kostenkurven. Die durchgezogene Funktionskurve beschreibt die Kostenveränderung, die bei einer bestimmten Anzahl Fehl-Einlagerungen anfallen. Die Kostensteigerung bei der daraus resultierenden Verschlechterung des Lagerorganisationsgrades ist deutlich sichtbar. Über die gestrichelte Kurve werden die durch die Reorganisation anfallenden Kosten beschriebene. Die resultierende 
Funktion (in Strich-Punkt dargestellt) zeigt dann den optimalen Zeitpunkt für die Durchführung der Reorganisationsmaßnahme.

\section{$4 \quad$ Zusammenfassung und Ausblick}

Die dargestellte Methodik ermöglicht also die Berechnung von optimalen Reorganisationszyklen für manuelle Kommissioniersysteme. Anhand der funktional approximierten Zusammenhänge zwischen Artikelanordnung und Kommissioniersowie Nachschubleistung können die durch Umpackprozesse entstehenden Kosten quantifiziert werden.

Aktuell sind die hinterlegten Funktionen in Abhängigkeit von der Anzahl falsch eingelagerter Artikel formuliert. Die Integration der Zeit als Abhängigkeitsfaktor ist als nächster Schritt geplant.

Die Validierung der gesamten Methodik erfolgt am Beispiel eines großen Unternehmens im Lebensmitteleinzelhandel. Die bisherigen Ergebnisse bestätigen jedoch die simulierten und approximierten Zusammenhänge und zeigen die Funktionsfähigkeit der Methodik.

\section{Literatur}

[Abd94] Abdel-Hamid, A., Borndörfer, R. On the Complexity of the Storage Assignment Problem. Konrad-Zuse-Zentrum für Informationstechnik, Berlin, 1994

[Cha11] Chan, F., Chan, H.: Improving the productivity of order picking of a manual-pick and multi-level rack distribution warehouse thtough the implementation of class-based storage. Expert Systems with Applications. 38, 3, S. 2686 - 2700. 2011

[Doe04] Dörner, K., Reeh, M., Strauss, C., Wäscher, G.: Evaluation von Artikelanordnungsmustern in der Mann-zur-Ware-Kommissionierung. Working Paper 3 / 2004. Otto-von-Guericke-Universität, Magdeburg, 2004

[Gar05] Garfinkel, M.: Minimizing Multi-zone Orders in the Correlated Storage Location Assignment Problem. PhD-Thesis, School of Industrial and Systems Engineering; Georgia Institute of Technology, Georgia, 2005

[Gla05] Glass, M., Marquardt, H.: Forschungsbericht FE 179: Schnellläuferstrategien in Lagern und dynamische Zonierung. TU Dresden, Institut für Fördertechnik, Baumaschinen und Logistik, Dresden, 2005

[Gra92] Gray, A., Karmarkar, U. S., Seidmann, A.: Design and operation of an order-consolidation warehouse: Models and application. European Journal of Operational Research, 58, S- 14 - 36. 1992 
[Gu05] Gu, J.: The forward reserve warehouse sizing and dimensioning problem. Dissertation Georgia Institute of Technology : 2005

[Gu07] Gu, J., Goetschalckx, M., McGinnis, L. F.: Research on warehouse operation: A comprehensive review. European Journal of Operational Research. 177, 1, S. 1 - 21. 2007

[Kos07] de Koster, R., Le-Duc, T., Roodbergen, K.: Design and control of warehouse order picking: A literature review. European Journal of Operational Research, 182, 2, S. 481 - 501. 2007

[Luo09] Luo, J., Tang, L., Hu, Y.: Heuristic Algorithm with Oscillation Strategy for a New Class of Assignment Problem. Systems Engineering - Theory \& Praxis. 29, 1, S.111 - 117. 2009

[Mup08] Muppani, V., Adil, G.: Class-based storage-location assignment to minimise pick travel distance. Internationale Journal of Logistics Research and Applications. 11, 4, S. 247 - 265. 2008

[Pet99] Petersen II, C., Schmenner, R.: An Evaluation of Routing and Volumebased Storage Policies in an Order Picking Operation. Decision Sciences. 30, 2, S. 481 - 501. 1999

[Pet99b] Petersen II, C.: The impact of routing and storage policies on warehouse efficiency. International Journal of Operations \& Production Management. 19, 10, S. 1053 - 1064. 1999

[Rat99] Ratliff, D., Rosenthal, A.: Order-Picking in a Rectangular Warehouse: A Solvable Case of the Traveling Salesman Problem. Operations Research. 31, 3, S. $507-521.1983$

[Ren08] Renaud, J., Ruiz, A: Improving product location and order picking activities in a distribution centre. Journal of the Operational Research Society. 59, 12, S. 1603 - 1613. 2008

[Rou00) Rouwenhorst, B., Reuter, B., Stockrahm, V., van Houtum, G. J., Mantel, R. J., Zijm, W.: Warehouse design and control: Framework and literature review. European Journal of Operational Research, 122, 3, S. 515 - 533. 2000

[Str10] Strack, G., Pochet, Y.: An Integrated Model for Warehouse and Inventory Planning. European Journal of Operational Research, 204, 1, S. 35- 50. 2010

[Vei97] Veitinger, M.: Controlling von Reorganisationsprozessen in der Logistik. Europäische Hochschulschriften: Reihe 5, Band 2182; Dissertation Technische Universität München, 1997

[Vic97] Vickson, R., Fujimoto, A.: Optimal storage locations in a carousel storage and retrieval problem. Location Science. 4, 4, S. 237 - 245. 1997 
[Wäs04] Wäscher, G.: Order picking: A survey of planning problems and methods. In: Dyckhoff, H.; Lackes, R. und Reese, J.. Supply Chain Management and Reverse Logistics. Springer-Verlag, Berlin, 2004 